\title{
DISPOSITIVOS DE ASISTENCIA PARA LA MOVILIDAD EN PERSONAS CON DISCAPACIDAD VISUAL: UNA REVISIÓN BIBLIOGRÁFICA
}

\author{
Ahmed Alejandro Cardona Mesa ${ }^{1}$, Ruben Dario Vasquez Salazar² \\ ${ }^{1}$ Ingeniero en Instrumentación y Control, estudiante de Maestría en Ingeniería - Gestión de la Automatización. \\ ahmed_cardona04072@elpoli.edu.co \\ 2 Magíster en Ingeniería - Automatización Industrial. Profesor Asociado, Facultad de Ingeniería. \\ rdvasquez@elpoli.edu.co \\ 1,2 Politécnico Colombiano Jaime Isaza Cadavid, Medellín, Colombia.
}

\section{RESUMEN}

Este documento presenta una revisión literaria de los dispositivos de ayuda en la movilidad para personas con discapacidad visual, con el objetivo de obtener una visión clara sobre el progreso de la tecnología y técnicas empleadas para asistir a esta población. De esta forma, se pretende obtener pautas básicas para analizar los equipos más relevantes, para ayudar a las personas con visión reducida y destacar las mejoras que se podrían implementar. Los dispositivos más comunes corresponden a la integración de diferentes sensores y componentes electrónicos en bastones, para aumentar su capacidad de detección de obstáculos. Además, se han desarrollado dispositivos con cámaras, que incluyen algoritmos de visión por computador y técnicas de Inteligencia Artificial, para mejorar los resultados y eficiencia de los equipos. Finalmente, se presentan las características fundamentales para los sistemas de asistencia, encontrando que aún no se cuenta con dispositivos que satisfagan las necesidades de los usuarios.

Palabras clave: Discapacidad visual, Tecnologías de asistencia, Visión por computador, Inteligencia Artificial.

Recibido: 12 Marzo, 2019. Aceptado: 26 de Abril 2019

Received: March 12, 2019 Accepted: April 26, 2019

\section{ASSISTANCE DEVICES FOR MOBILITY IN VISUALLY-IMPAIRED PEOPLE: A BIBLIOGRAPHIC REVIEW}

ABSTRACT

This document presents a literary review of the different mobility aids for people with visual disabilities, with the aim of obtaining a clear vision on the progress of technology and techniques used for assistance in this population. In this way, it is intended to obtain basic guidelines to analyze the most relevant equipment found in the literature, to help people with reduced vision and highlight the improvements that could be implemented with this purpose. The most common devices correspond to the integration of sensors and electronic components in canes, to increase their ability to detect obstacles. In addition, devices with cameras have been developed, including computer vision algorithms and artificial intelligence techniques, to improve the results and efficiency of the equipment. Finally, the fundamental characteristics that systems of assistance to this population must have are presented, finding that there is not yet a device that meets the needs of the users.

Keywords: Visual disability, Assistive technologies, Computer vision, Artificial Intelligence.

Cómo citar este artículo: A. Cardona, R. Vasquez, "Dispositivos de asistencia para la movilidad en personas con discapacidad visual: una revisión bibliográfica", Revista Politécnica, vol. 15, no.28 pp. 107-116, 2019. DOI: https://doi.org/10.33571/rpolitec.v15n28a10 


\section{INTRODUCCIÓN}

A nivel mundial existen 39 millones de personas que son completamente ciegas y 246 millones que tienen baja visión, siendo un total de 285 millones de personas con discapacidad visual. Más de la mitad de esta población sobre pasan los 50 años de edad y viven en condiciones de pobreza. Los errores de refracción no corregidos y las cataratas, son las dos causas más comunes de discapacidad visual, en la Fig. 1, se presentan las cifras porcentuales de las principales patologías que ocasionan ceguera a nivel mundial [1].

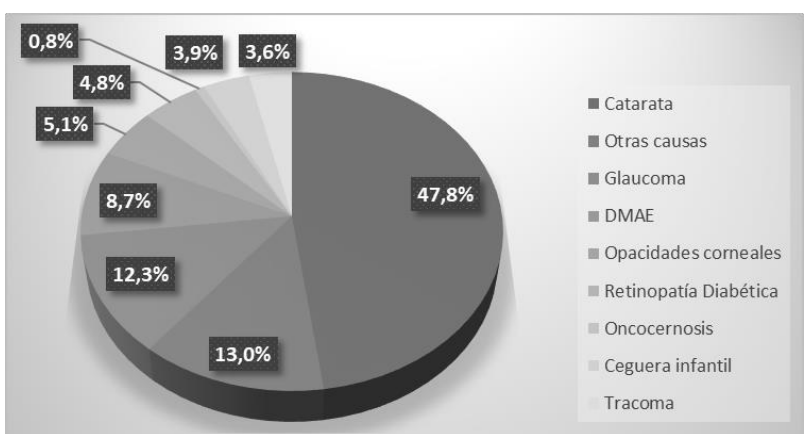

Fig 1. Cifras de las principales causas de ceguera a nivel mundial.

En un estudio basado en 15 encuestas de países latinoamericanos, sobre la prevalencia de discapacidad visual funcional, esta se ubicó entre 0.9 a $2.2 \%$ de la población mayor de 50 años, las causas principales de baja visión corresponden a degeneración macular relacionada con la edad (26\%), glaucoma (23\%), retinopatía diabética $(19 \%)$, otras patologías de segmento posterior (15\%), opacidades de la córnea (7\%), y las complicaciones de la cirugía de cataratas (4\%), de acuerdo a las estadísticas, alrededor de 4.000 personas por cada millón, necesitan servicios de baja visión funcional [2].

En todo el territorio Colombiano, se estima que hay 7.000 personas ciegas por cada millón (más de 296.000 habitantes). Estas cifras corresponden a toda la población del país, aunque el impacto negativo en el aprendizaje y la desadaptación en la población infantil es mayor, encontrando que aproximadamente el $2 \%$ de los niños en edades entre 6 y 11 años presentan una agudeza visual menor a $20 / 60$ y el $75 \%$ corrigen con el uso de lentes formulados, lo cual indica que el mayor porcentaje de las causas son defectos de refracción y alteraciones visuales que pueden corregirse en alguna proporción, con la fórmula óptica adecuada [3].

La Organización Mundial de la Salud (OMS) estima que para el año 2020, la ceguera y la discapacidad visual grave se constituirán en un problema de salud pública para el mundo. Esta situación generará una carga social, económica y cultural de alcance universal, con énfasis en los países de ingresos medianos y bajos [1]. Aunque existen ayudas para mejorar la visión residual en personas con discapacidad visual leve a moderada, estas no sirven para personas con discapacidad visual severa a profunda (DVS, DVP) o con ceguera, las cuales necesitan desarrollar habilidades que sustituyan la visión [4]. Para ellas el logro de la independencia en actividades básicas e instrumentales de la vida diaria es más difícil, teniendo en cuenta que puede coexistir otro tipo de discapacidades que limitan aún más este proceso.

Las personas con discapacidad visual son muy dependientes de otras o de algunas herramientas, para poder desplazarse de forma segura en ambientes desconocidos. Generalmente, dependen de herramientas como el bastón o los lazarillos, cada una de estas ayudas tiene sus propias limitaciones. El bastón tiene un alcance aproximado de 1.5 metros y no sirve para detectar obstáculos sobre el nivel de la cintura. En el caso de los lazarillos, se requiere de mucho tiempo para entrenar a los perros, también hay un tiempo adicional para que la persona se adapte a estos y su cuidado apropiado se dificulta para las personas con discapacidad visual [5].

La discapacidad visual tiene un muy alto impacto en la calidad de vida de los individuos, incluyendo en las habilidades para trabajar y para desarrollar relaciones personales. La pérdida de visión causa que el individuo pierda la percepción espacial aproximadamente en un $90 \%$. Casi la mitad de las personas con discapacidad visual se sienten moderada o completamente apartadas de las personas y cosas que los rodean. Para que se pueda producir una verdadera integración de las personas con discapacidad visual a la sociedad, deben erradicarse las barreras físicas, tecnológicas y mentales que puedan existir debido a sus discapacidades, porque como cualquier ciudadano, ellos tienen funciones sociales a partir del desarrollo de sus aptitudes y capacidades personales [6].

Los recientes avances tecnológicos, tienen como objetivo, ayudar y mejorar la calidad de vida de las 
personas con discapacidad visual, aumentando su independencia en el desarrollo de sus actividades diarias. Dispositivos electrónicos basados en diferentes clases de sensores integrados en bastones, para aumentar la efectividad de detección de obstáculos y percepción espacial de las personas con esta discapacidad, sistemas de cámaras con algoritmos de visión por computador y la implementación de técnicas de inteligencia artificial (IA), para la lectura de textos, el reconocimiento de rostros, entre otros, son las principales tecnologías que se ponen a disposición de las personas con visión reducida $[7,8]$.

Los avances tecnológicos no han llegado a todos por igual. En un mundo en el que el siguiente gran desarrollo se presenta a cada minuto, principalmente en el área de software y dispositivos móviles, las personas con determinada discapacidad visual han quedado fuera de plano. Sin embargo, algunas herramientas innovadoras tratan de utilizar el potencial de las nuevas tecnologías para hacerles la vida más fácil [9]. En este documento se realiza una revisión de literatura, que presenta los diferentes tipos de proyectos tecnológicos, que se han desarrollado como asistencia para la movilidad y navegación autónoma de personas con discapacidad visual.

La metodología y estructura de este documento de revisión consiste en las siguientes etapas: el capítulo 2 se realiza una contextualización de la definición de las tecnologías de asistencia, especialmente para personas con discapacidad visual; posteriormente en el capítulo 3 se realiza una búsqueda en fuentes bibliográficas académicas e investigativas a través de bases de datos especializadas en ingeniería (principalmente IEEE y ScienceDirect) y metabuscadores académicos (principalmente GoogleScholar y SemanticScholar); estos últimos arrojan resultados de diversas fuentes que deben ser consultadas particularmente. En este paso, se seleccionan únicamente los artículos que realicen un aporte o presenten una innovación, concepto o desarrollo que pueda verse reflejado en el impacto en el desempeño de actividades cotidianas por parte de personas con alguna discapacidad visual, buscando encontrar las características más relevantes que generan un impacto en este tipo de asistencia. Posteriormente se abstrae esta información y se agrupa en diferentes afinidades tecnológicas de modo que se aborden las temáticas en que se pueden categorizar las tecnologías encontradas; en el capítulo 4 se realiza una síntesis de los hallazgos realizados con el fin de identificar posibles líneas de trabajo y especificidades necesarias en el desarrollo de un futuro dispositivo para esta tarea de asistencia; finalmente en el capítulo 5 se presentan las conclusiones.

\section{TECNOLOGÍAS DE ASISTENCIA}

Según el Consejo Europeo de Optometría y Óptica [10], la baja visión corresponde a una anomalía visual que dificulta la capacidad de realizar tareas cotidianas. Esta condición no puede ser corregida con fórmulas ópticas o intervención médica. Síntomas típicos de anomalía visual son la pérdida de agudeza y campo visual, la pérdida de sensibilidad al contraste, escaza visón nocturna, problemas con el reconocimiento de colores, así como el aumento de la sensibilidad a la luz (como deslumbramiento o fotofobia). Por otro lado, El Código Internacional de Enfermedades (CIE-10) define la ceguera como "una agudeza visual menor de $0.05(20 / 400,3 / 60,1.3$ logMAR), o una correspondiente pérdida del campo visual menor de 10 grados en el mejor ojo con la mejor corrección posible". Finalmente, la discapacidad visual severa comprende una agudeza visual por debajo de $20 / 200,6 / 60$ e igual o superior a 20/400 o 3/60, y por discapacidad visual leve, una agudeza visual entre $20 / 60$ o 6/18 y 20/200 o 6/60 [11].

La orientación y desplazamiento autónomo, es una actividad directamente relacionada con el sentido de la visión, por esta razón, la dificultad en la movilidad de personas con discapacidad visual, se convierte en el problema más común que deben afrontar en sus actividades diarias. Por este motivo, se hace indispensable que esta población recurra a dispositivos y técnicas que sirvan como ayuda en el desarrollo de sus vidas cotidianas [12]. La herramienta más utilizada por las personas en condición de discapacidad visual, es el bastón blanco, el cual sirve de guía para desplazarse de manera autónoma por diferentes lugares, éste instrumento tiene tres características básicas: distintivo, protección e información [13]. Algunos de los esfuerzos más recientes en cuanto a tecnologías asistenciales para personas con discapacidad visual, apuntan a proveer dispositivos electrónicos que se adapten a las tareas de movilidad y desplazamiento con el fin de facilitarlas [14].

2.1 Ayudas electrónicas integradas en bastones. La tecnología asistida es cualquier equipo o sistema modificado, que se utiliza para, mejorar las 
habilidades funcionales de personas con algún tipo de discapacidad funcional [15]. En el caso de las personas con baja visión, se han desarrollado bastones electrónicos con sensores, ultrasónicos, que permiten detectar obstáculos cercanos con anticipación.

Se realiza una consulta en bases de datos y metabuscadores con las siguientes palabras claves y fórmulas:

- Cane AND Electronic Aid

- Cane AND Assistance technology

- Visual disability AND electronic cane

- Ultrasound AND visual disability

- Smart Cane AND Electronic Aid.

Basados en la emisión de un pulso de ultrasonido y midiendo el tiempo transcurrido entre la emisión del sonido y la recepción del eco, se puede determinar la distancia a la que se encuentra el obstáculo que produce la reflexión de la onda sonora [16-18]. Estos dispositivos presentan alertas auditivas o vibratorias, para prevenir al usuario sobre la proximidad de un objeto y se encuentran desarrollos similares con la implementación de sensores de proximidad [19], además de variaciones estructurales, sustituyendo la adaptación de los sensores en el bastón por el uso de elementos como sombreros y chalecos bajo el mismo principio. Diferentes aplicaciones proponen aumentar la funcionalidad de estos dispositivos, con el uso de una serie de tecnologías, entre las que se incluyen sensores infrarrojos para detectar cambios de nivel en terrenos irregulares, GPS (Global Positioning System), GSM (Global System for Mobile Communications), GPRS (General Packet Radio Service) para realizar el posicionamiento de las personas, logrando ubicarlos en un lugar determinado o guiarlos hasta una dirección específica, RFID (Radio-Frequency Identification) para mejorar la navegación en zonas con diferentes etiquetas, ubicadas en lugares estratégicos, entre otros. Finalmente, se integran diversas tecnologías y herramientas, de acuerdo a las necesidades de la aplicación, para mejorar la asistencia en la movilidad y ubicación de personas con discapacidad visual [20-24].

Uno de los proyectos más importantes relacionado con la implementación de componentes electrónicos y sensores, corresponde al producto Smart Cane, desarrollado para detectar los objetos en el camino y producir instrucciones para la orientación [25]. El Smart Cane es un dispositivo portable que cuenta con un sistema de sensores ultrasónicos, un sensor de humedad, un microcontrolador, una serie de motores y un zumbador para detectar los obstáculos en frente del usuario y poder proporcionar instrucciones a través de mensajes de audio o alertas vibratorias en la mano. La mayoría de los dispositivos electrónicos desarrollados para la asistencia de personas con discapacidad visual, dependen de la información obtenida del entorno y los datos se transmiten al usuario a través de dispositivos de audio o alertas vibratorias. Se encuentran diferentes opiniones sobre cuál es el mejor tipo de retroalimentación, aunque sigue siendo un tema de discusión que solo puede ser resuelto por el usuario final de los diferentes sistemas implementados.

Los dispositivos electrónicos de apoyo a la movilidad, no han tenido gran éxito comercial ya que resulta bastante difícil competir con la sencillez y bajo costo del bastón. Prueba de esto es que, muchos de estos equipos que en algún momento fueron comercializados, actualmente se encuentran por fuera del mercado [26]. Estas ayudas para la asistencia en la movilidad, basadas en la implementación de sensores para la detección de obstáculos cercanos, sufren de algunas falencias de diseño. La más importante se encuentra relacionada con la interfaz de comunicación, que transmite escasa información al usuario, sin especificar el tipo de objeto que es detectado y que puede representar un peligro.

\subsection{Ayudas a partir de algoritmos de Visión Artificial.}

La visión artificial es una disciplina que pretende simular los diferentes procesos y elementos que otorgan visión a una máquina. Estas incluyen las propiedades geométricas y otras, como el color, iluminación, textura y composición. La visión, tanto para un hombre como para un computador. Esta disciplina consta principalmente de dos fases: adquirir una imagen e interpretarla. El proceso de adquisición de imágenes, se realiza por medio de una cámara. De esta forma, solo resta implementar herramientas para interpretar las imágenes, distinguir los objetos de la escena, extraer información de ellos y resolver aspectos más particulares según las necesidades que se desean satisfacer [27, 28].

La visión por computador como sustituto de la visión humana, es una herramienta fundamental en la implementación de dispositivos de apoyo a personas 
con discapacidad visual. Algunas de las tareas principales para las cuales se utiliza la visión artificial como apoyo a personas con discapacidad visual con buenos resultados, se encuentra la asistencia en la movilidad, orientación, reconocimiento de objetos, acceso a información impresa e interacción social. La visión artificial, a diferencia de las tecnologías que incorporan dispositivos electrónicos con sensores, permite una interpretación del entorno, ofreciendo un mayor grado de representación de la realidad a partir de mayor complejidad en el procesamiento de la información. En los últimos años, se ha extendido el uso de la visión artificial para apoyar a estas [29-31] con prototipos basados en computadores portátiles.

En los últimos años, la integración de cámaras digitales en los Smartphones, ha dado inicio a una nueva generación de herramientas que permiten a estas personas, realizar tareas cotidianas como la detección de obstáculos [32], leer material impreso [33], reconocer objetos genéricos en supermercados $[34,35]$, orientarse espacialmente en lugares interiores o exteriores [36] e interactuar socialmente con las personas que los rodean [37].

Se realiza una consulta en bases de datos y metabuscadores con las siguientes palabras claves y fórmulas:

- Computer Vision AND Assistance
technology
- Computer Vision AND Visual disability
- Digital Image Processing AND Visual
disability

Un ejemplo de un sistema de detección de obstáculos en tiempo real basado en algoritmos de visión artificial se presenta en [38] para la detección de obstáculos y la asistencia en la movilidad de personas con discapacidad visual, en interiores y exteriores. Esta aplicación funciona con la ayuda de un dispositivo móvil con cámara. El equipo puede detectar objetos estáticos y dinámicos en una secuencia de video. La implementación del sistema en el teléfono inteligente constituye una gran ayuda para la movilidad de la población con discapacidad visual, ya que los smartphones son cada vez más portables y con mayor capacidad de procesamiento. Sin embargo, la detección de objetos en lugares con poca iluminación y aquellos que están en constante movimiento, no pueden ser detectados de forma eficiente. Además, los resultados de la aplicación pueden variar considerablemente, en base a la calidad de los videos que puede adquirir la cámara del teléfono móvil.
Aunque la visión artificial se ha convertido en una herramienta muy utilizada para el desarrollo de tecnologías de asistencia para la población con discapacidad visual, todavía no se alcanza todo el potencial que puede ofrecer, aprovechando los actuales desarrollos en hardware, con la aparición de procesadores de gran capacidad para implementar los algoritmos en software [39]. Un problema común en el desarrollo de nuevas tecnologías, es que no son diseñadas con base en las necesidades específicas de las personas en esta condición, por esta razón, se desconocen los verdaderos requerimientos de los usuarios, causando que los dispositivos desarrollados no obtengan los resultados deseados. Otro problema significativo es que los dispositivos presentan un gran tamaño y resultan difíciles de usar.

\subsection{Ayudas integrando Visión por Computador y Redes Neuronales Artificiales (RNA).}

El concepto de Inteligencia Artificial puede tener diferentes connotaciones, dependiendo de la fuente que se tome como referencia y del punto de vista de esta. Lo que sí es claro, es que la IA tiene como uno de sus objetivos, el estudio del comportamiento inteligente de las máquinas [40]. En otras palabras, la Inteligencia Artificial es el estudio de cómo hacer que los ordenadores, hagan cosas que por el momento son realizadas mejor por los seres humanos [41].

Las redes neuronales fueron creadas dentro del movimiento de investigación en Inteligencia Artificial. Surgiendo como la posibilidad de poder realizar tareas cotidianas para un ser humano con la ayuda de máquinas, algunas de las tareas más comunes son: el reconocimiento de patrones, reconocimiento de caracteres, reconocimiento de voz, reconocimiento de rostros, reconocimiento de expresiones faciales, la planificación de diferentes tareas, entre otras. En un artículo de 1950 con título "Computing machinery and intelligence", Alan Turing se pregunta si las máquinas pueden pensar [42]. Como un acercamiento a la respuesta, propone una prueba denominada el test de Turing, para lograr determinar si una máquina puede simular una conversación humana: "un usuario dialogando mediante un canal escrito debe determinar si su interlocutor es un ser humano o una máquina" [43].

Uno de los mayores retos que el hombre se propuso es el de comprender el funcionamiento de los órganos de los sentidos, para posteriormente tratar 
de imitar dicho funcionamiento por medio de las tecnologías existentes, buscando así la solución de problemas del mundo real [44]. De esta forma, se utilizan en conjunto, diferentes algoritmos de visión por computador para el procesamiento de imágenes y técnicas de inteligencia artificial para el análisis de datos y la obtención final de los resultados.

Se realiza una consulta en bases de datos y metabuscadores con las siguientes palabras claves y fórmulas:

- Neural Network AND Assistance technology

- Artificial Neural Networks AND Visual disability

- Artificial Intelligence AND Visual disability

Diversas aplicaciones para la asistencia de personas con discapacidad visual integran el uso de sistemas entrenados con RNA y la adquisición de imágenes con cámaras para el reconocimiento de patrones $[45,46]$. La detección de diferentes tipos de objetos como puertas, esquinas, bordes, caminos, entre otros que facilitan el reconocimiento de obstáculos [47-49] y el reconocimiento de rostros y expresiones faciales para mejorar las interacciones sociales [50].

\subsection{Ayudas con procesos de clasificación de imágenes usando Deep Learning.}

Machine Learning es en realidad un conjunto de técnicas que hacen parte de la inteligencia artificial que, basadas en algoritmos, buscan el aprendizaje dentro de grandes conjuntos de datos. Una característica muy importante de estos algoritmos es la predicción de nuevos casos, basándose en la experiencia aprendida del conjunto de datos utilizados para su entrenamiento [51].

El Deep Learning (DL) o aprendizaje profundo, es una rama del Machine Learning con una familia de algoritmos que simulan el proceso realizado por las neuronas cerebrales, para llevar a cabo el reconocimiento de voz, imágenes, palabras, entre otras aplicaciones. Dichos algoritmos funcionan en un proceso por capas, y en cada capa se encuentran neuronas que realizan un procesamiento simple sobre las salidas de la capa de neuronas anterior. Sin embargo, no conviene llevar demasiado lejos la analogía con las estructuras que se pueden encontrar en un cerebro real, ya que las neuronas artificiales son mucho más simples que las naturales. Se puede asumir que las redes neuronales artificiales constituyen modelos matemáticos con parecido superficial a las redes naturales, que realizan funciones útiles porque pueden adaptarse o aprender en cierto sentido [52]. El Deep Learning es un concepto que surge de la idea de imitar el cerebro a partir del uso de hardware y software, para crear una inteligencia artificial utilizando una capacidad de abstracción jerárquica, es decir, una representación de los datos de entrada en varios niveles [53]. A partir de esto, se ha desarrollado un rápido crecimiento en la cantidad de arquitecturas y algoritmos de entrenamiento [54].

Para la implementación del DL se utilizan especialmente, estructuras que se aproximan a la distribución del sistema nervioso de los seres vivos teniendo grupos de neuronas artificiales que realizan la tarea de detectar ciertas características existentes en los objetos presentes en las imágenes. Las aplicaciones que incorporan algoritmos de visión artificial, son unas de las áreas donde el DL proporciona una mejora significativa con respecto a técnicas más tradicionales.

Se realiza una consulta en bases de datos y metabuscadores con las siguientes palabras claves y fórmulas:

- Deep Learning AND Assistance technology

- Convolutional Neural Networks AND Visual disability

- Object Detection AND Automatic AND Visual disability

- Image Classification AND Assistance Technology

Los algoritmos de DL se convierten en una nueva alternativa para el desarrollo de sistemas que permitan el reconocimiento de expresiones corporales, la clasificación de imágenes a partir de grandes bases de datos [55, 56], la implementación de robots móviles evasores de obstáculos, cuyo algoritmo y principio de funcionamiento puede ser adaptado para la asistencia en la movilidad autónoma de personas con visión reducida, para la detección y evasión de objetos $[57,58]$ y la clasificación y categorización de obstáculos [59].

\section{ANÁLISIS}

La cantidad de referencias encontradas en cada uno de los tópicos definidos en el capítulo anterior, dan cuenta del desarrollo histórico de trabajo que ha tenido cada uno de ellos (Fig. 2). De allí se analiza que el tópico con más desarrollo fueron las ayudas electrónicas con un $43 \%$, seguramente debido a que es una tecnología que existe hace décadas y, en contraste con la visión artificial, por ejemplo, apenas 
tiene alto desarrollo significativo e investigación desde las últimas décadas.

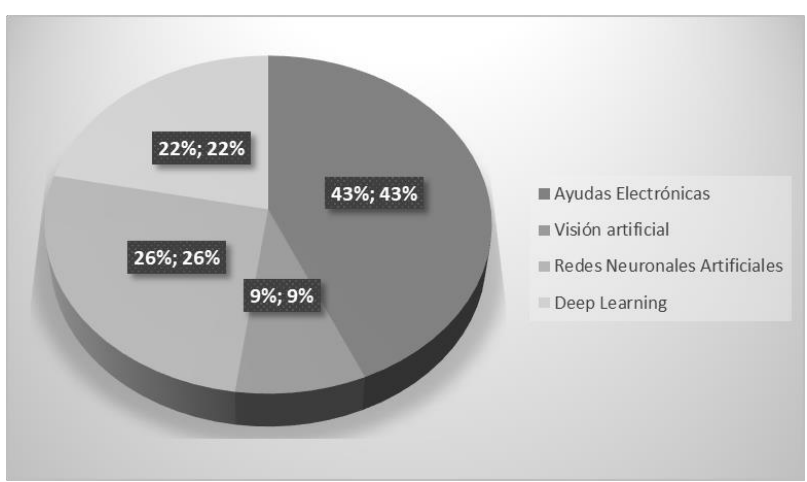

Fig. 2. Porcentaje de referencias encontradas en cada tópico.

Los diversos dispositivos de ayuda para las personas con discapacidad visual deben proporcionar una serie de características que garanticen su funcionalidad de asistencia para el usuario, estas corresponden a una interfaz de retroalimentación clara y sencilla para las personas, un correcto desempeño bajo diferentes ambientes y condiciones de iluminación, la capacidad de detectar objetos estáticos y dinámicos para analizar cualquier aparición repentina de un obstáculo en el camino y la integración de hardware y software con el rendimiento adecuado para el procesamiento de los datos. Estas características son básicas y fundamentales para el diseño e implementación de un dispositivo de asistencia para personas con discapacidad visual, ya que cada característica tiene un impacto significativo en el desempeño del equipo.

Ninguna de las aplicaciones encontradas presenta un funcionamiento completamente satisfactorio en función de las características básicas definidas y de las necesidades de los usuarios para la asistencia en su movilidad. Es destacable el hecho de que cada prototipo desarrollado presenta funciones especiales ante los demás y podría tener funciones adicionales, pero ninguno de ellos admite todas las características necesarias para ser considerado un servicio integral de ayuda a las personas con discapacidad visual. Esto significa que no es posible considerar a ninguno de los desarrollos como un dispositivo ideal. Los principales motivos para estas limitantes corresponden a que la mayoría de los desarrolladores e investigadores centran sus esfuerzos para la implementación de una técnica diferente para proporcionar una nueva característica, pero no prestan atención en que sus trabajos admitan características fundamentales, antes de agregar otras nuevas, con el propósito de crear dispositivos más innovadores desde una perspectiva académica que funcionales como elementos de asistencia y servicio para los usuarios. Otra razón por la que se presentan estas limitaciones es que los diseñadores no realizan una investigación adecuada que permita establecer de forma clara y concisa cual es la percepción y requerimientos fundamentales de personas con esta condición, acerca de un sistema que brinde asistencia en las actividades de su vida cotidiana.

\section{CONCLUSIONES}

En el mundo se han realizado diferentes desarrollos e investigaciones, alrededor de la solución de problemas para personas con discapacidad visual. A pesar de los esfuerzos, no se ha logrado integrar las herramientas adecuadas que permitan proporcionar una asistencia confiable que satisfaga las principales necesidades de esta población.

Para desarrollar un dispositivo para la asistencia en la movilidad de personas en condición de discapacidad, es necesario realizar una jerarquización de las principales necesidades que tienen estas personas, debido a la gran cantidad de información que ofrece el medio ambiente y la capacidad de procesamiento de los dispositivos electrónicos. Este estudio es vital antes de definir las características técnicas de un equipo de asistencia.

El uso de técnicas de inteligencia artificial, en conjunto con herramientas de visión por computador, debe realizar una integración de hardware y software que permita la clasificación de objetos y detección de obstáculos, analizados en video en tiempo real, generando alertas posiblemente auditivas para los usuarios en función del orden jerárquico pre-establecido.

\section{REFERENCIAS BIBLIOGRÁFICAS}

[1] «World Health Organization,» World Health Organization, 2017. [En línea]. Available: http://www.who.int/features/factfiles/blindness/blind ness_facts/en/. [Último acceso: 1309 2017].

[2] Y. Z. Cañón, «La baja visión en Colombia y en el mundo, » cien. tecnol. salud. vis. ocul, vol. 9, no 1, pp. 117-123, 2011. 
[3] H. Linburg, R. Espinoza, V. G. Lansingh y J. C. Silva, «Functional low vision in adults from Latin America: findings from population-based surveys in 15 countries,» Panam Salud Publica 37(6), pp. 371378, 2015.

[4] E. O’Brien, A. Mohtar, L. Diment y K. Reynolds, «A Detachable Electronic Device for Use With a Long White Cane to Assist With Mobility,» Taylor Francis OnLine, pp. 219-226, 2014.

[5] M. S. Quintana, "Áreas específicas para la atención de personas con discapacidad visual,» Secretaria de Educación y Cultura, Chihuahua, 2011.

[6] Y. S. Pascuas, E. O. Vargas y M. Sáenz, "Tecnologías de la información y las comunicaciones para personas con necesidades educativas especiales ${ }^{*}, »$ Entramado, vol. 11, nํ 2, pp. 240-246, 2015.

[7] J. A. Ortiz, "Accesibilidad tecnológica para limitados visuales en las bibliotecas públicas mayores en la ciudad de Bogotá.,» Universidad de la Salle, Bogotá D.C., 2009.

[8] H. Escobar, C. Vélez y C. Barrera, «Ayudas externas para mejorar la independencia en personas con discapacidad visual,» Revista Cubana de Oftalmología, pp. 1-17, 2015.

[9] A. Semenoy, «Las tecnologías de la información y la comunicación en la enseñanza,» UNESCO, Moscú, 2005.

[10] «Documento de posición oficial - Baja Visión,» European Council of Optometry and Optics - ECOO, Madrid, 2011.

[11] «Definitions of blindness and visual impairment," Organización Mundial de la Salud (OMS), Ginebra, 2012.

[12] «Orientaciones pedagógicas para la atención educativa a estudiantes con discapacidad motora,» Ministerio de Educación Nacional, Bogotá, 2006.

[13] «Investigación en Tecnologías de Inteligencia Ambiental para la Salud del Futuro,» Instituto de Salud Carlos III, Madrid, 2009.

[14] V. García, "Auxiliares de movilidad en la discapacidad visual. Terapia asistida por perros,» Universidad de Valladolid, Valladolid, 2016.

[15] O. Hernández, «Tecnología Asistida: Propuesta de terapia ocupacional para aumentar el desempeño ocupacional de un usuario con parálisis cerebral,» Revista Chilena de Terapia Ocupacional, vol. 13, no 2, pp. 33-43, 2013.

[16] A. Egaña, R. Ceres, F. Seco y J. L. Pons, «Detección ultrasónica de obstáculos por medida diferencial para la movilidad de invidentes,» de XXV Jornadas de Automática, Ciudad Real, 2004.
[17] R. Ávila, «Diseño y construcción de un bastón con sistema ultrasónico producido por un microcontrolador PIC16F84 para niños invidentes y débiles visuales,» Instituto Politécnico Nacional, México D.F., 2015.

[18] J. D. Alvarado y V. Mosquera, Visión Electrónica, vol. 10, № 1, pp. 1-6, 2016.

[19] J. L. Rubio, C. Solon, R. Bayan y M. Barrio, «Robótica y UX para la Mejora de las Interacciones en el Espacio Urbano: Desarrollo y Perfeccionamiento de un Bastón Electrónico para Invidentes,» de IX Congreso Internacional Ciudades Creativas, Madrid, 2014.

[20] L. Nieto, C. Padilla y M. Barrios, «Design and implementation of electronic AID to blind's cane," IEEE, pp. 932-935, 2014.

[21] J. Faria, S. Lopes, H. Fernandes, P. Martins y J. Barroso, «Electronic white cane for blind people navigation assistance,» de World Automation Congres, Porto, 2010.

[22] E. SathyaNarayanan, D. Gokul Deepan, B. P. Nithin y P. Vidhyasagar, "loT Based Smart Walking Cane For Typhlotic with Voice Assistance,» de 2016 Online International Conference on Green Engineering and Technologies, Combatore, India, 2016.

[23] W. F. Ramírez, «Sombrero electrónico con ultrasonido para la orientación de personas con discapacidad visual,» Inclusión \& Desarrollo, vol. 3, no 2, pp. 92-96, 2016.

[24] A. Wahab, M. Helmy, A. Talib, H. Kadir, A. Johari, A. Noraziah, R. Sidek y A. Mutalib, «Smart Cane: Assistive Cane for Visually-impaired People,» Comput. Sci, vol. 8, no 4, 2011.

[25] S. Bharambe, R. Thakker, H. Patil y K. Bhurchandi, «Substitute Eyes for Blind with Navigator Using Android,» Proceedings of the India Educators Conference (TIIEC); pp. 38-43, 2013.

[26] U. Roentgen, G. Gelderblom, M. Soede y L. de Witte, «Inventory of Electronic Mobility Aids for Persons with Visual impaimente A Literature Review, 》 Journal of Visual Impairment \& Blindness, vol. 102, no 11, 2008.

[27] E. A. Sobrado, «Sistema de visión artificial para el reconocimiento y manipulación de objetos, usando un brazo robot., » Pontificia Universidad Católica del Perú, Lima, 2003.

[28] A. González, F. Martínez, A. Pernia, F. Alba, M. Castejón, J. Ordieres y E. Vergara, Técnicas y Algoritmos Básicos de Visión Artificial, La Rioja: Universidad de la Rioja, 2006.

[29] J. Liu y X. Sun, «A Survey of Vision Aids for the Blind, $\gg$ 6th World Congress on Intelligent Control and Automation, vol. 1, pp. 4312-4316, 2006. 
[30] R. Manduchi y J. Coughlan, «Computer) vision without sight,» Communications of the ACM, vol. 55, no 1, pp. 96-104, 2012.

[31] S. Carrato, S. Marsi, F. Medvet, F. Pellegrino, G. Rampour y M. Vittori, «Computer vision for the blind: a dataset for experiments on face detection and recognition, » MIPRO, pp. 1206-1211, 2016.

[32] P. Vera, D. Zenteno y J. Salas, «A SmartphoneBased Virtual White Cane,» de Pattern Analysis and Applications, 2013.

[33] P. Lanigan, A. Paulos, A. Williams, D. Rossi y P. Narasimhan, «Trinetra: Assistive Technologies for Grocery Shopping for the Blind,» IEEE Trinetra: Assistive Technologies for Grocery Shopping Wearable Computers, pp. 147-148, 2006.

[34] S. Krishna y S. Panchanathan, "Assistive Technologies as Effective Mediators in Interpersonal Social Interactions for Persons with Visual disability,» Computers Helping People with Special Needs, pp. 316-323, 2010.

[35] «LookTel,» [En línea]. Available: http://www.looktel.com. [Último acceso: 2110 2017]. [36] «KNFB Reader,» 2012. [En línea]. Available: http://www.knfbreader.com. [Último acceso: 1710 2017].

[37] «Ciaffoni,» Ariadne GPS Mobility and map exploration for all, [En línea]. Available: http://www.ariadnegps.eu. [Último acceso: 2210 2017].

[38] R. Tapu, B. Mocanu y T. Zaharia, «A computer vision system that ensure the autonomous navigation of blind people,» IEEE, 2013.

[39] L. Pena y Y. Rodríguez, «Dispositivo apuntador mediante visión artificial, adecuado para usuarios de computador con discapacidad motora en miembros superiores., » Universidad Distrital Francisco José de Caldas, Bogotá, 2015.

[40] G. Choque, «Inteligencia Artificial, Perspectivas y realizaciones. Redes Neuronales.,» La Paz, 2002. [41] J. R. Salao, «Estudio de las Técnicas de Inteligencia Artificial mediante el apoyo de un software educativo,» Escuela Superior Politécnica de Chimborazo, Riobamba, 2009.

[42] A. M. Turing, "Computing Machinery and Intelligence,» Mind 49, pp. 433-460, 1950.

[43] C. Sarraute, "Aplicación de las Redes Neuronales al Reconocimiento de Sistemas Operativos, " Universidad de Buenos Aires, Buenos Aires, 2007.

[44] A. Cantero y E. Martínez, «Visión por computadora: identificación, clasificación y seguimiento de objetos.,» Universidad Nacional del Este, Ciudad del Este, 2014.
[45] P. P. García, «Reconocimiento de imágenes utilizando Redes Neuronales Artificiales,» Universidad complutense de Madrid, Madrid, 2013.

[46] C. Rocha y J. Escorcia, "Sistema de Visión Artificial para la Detección y el Reconocimiento de Señales de Tráfico basado en Redes Neuronales,» de Eighth LACCEI Latin American and Caribbean Conference for Engineering and Technology, Arequipa, 2010.

[47] X. Yang y Y. Tian, «Robust door detection in unfamiliar environments by combining edge and corner features,» IEEE Computer Society Conference on Computer Vision and Pattern Recognition, pp. 57-64, 2010.

[48] H. Mesa, J. Branch y C. López, «Sistema de detección de esquinas en imágenes digitales en tonos de gris, basado en redes neuronales artificiales,» Avances en Sistemas e Informática, vol. 5, № 3, pp. 195-200, 2008.

[49] M. Moreno, S. Shahrabadi, J. José, J. du Buf y J. Rodrigues, "Realtime local navigation for the blind: detection of lateral doors and sound interface,» Procedia Computer Science, vol. 14, pp. 74-82, 2012.

[50] X. Chen, X. Yang, M. Wang y J. Zou, "Convolution Neural Network for Automatic Facial Expression Recognition.,» IEEE2017, pp. 814-818, 2017.

[51] G. J. P. Restrepo, «Aplicación del Aprendizaje Profundo (Deep Learning) al Procesamiento de Señales Digitales,» Universidad Autónoma de Occidente, Santiago de Cali, 2015.

[52] A. A. Ortiz, «Algoritmo Multiclasificador con Aprendizaje Incremental que Manipula Cambios de Conceptos,» Universidad de Granada, Granada, 2014.

[53] L. Deng y D. Yu, «Deep Learning: Methods and Applications,» Foundations and Trends $₫$ in Signal Processing, vol. 7, no 34, pp. 197-387, 2014.

[54] R. Colombo, «Deep Learning para el reconocimiento de imágenes en Raspberry Pi 2,» Universidad de La Laguna, La Laguna, 2016.

[55] R. Ibáñez, A. Soria, A. Teyseyre y M. Campo, «Evaluación de técnicas de Machine Learning para el reconocimiento de gestos corporales," de 15th Argentine Symposium on Articial Intelligence, ASAI, Panamá, 2014.

[56] P. Morales, A. Pérez y G. Camps-Valls, "Remote Sensing Image Classification With LargeScale Gaussian Processes,» IEEE TRANSACTIONS ON GEOSCIENCE AND REMOTE SENSING, 2017.

[57] H. Yu, R. Hong, X. Huang y Z. Wang, «Obstacle Detection with Deep Convolutional Neural Network,» 
Sixth International Symposium on Computational Intelligence and Design, 2013.

[58] T. Ubbens y D. Shuurman, «Vision Based Obstacle Detection Using a Support Vector Machine,» IEEE, pp. 459-461, 2009.

[59] M. Poggi y S. Mattoccia, «A Wearable Mobility Aid for the Visually Impaired based on embedded 3D Vision and Deep Learning,» IEEE Workshop on ICT solutions for eHealth , 2016. 